\title{
Morphology-Dependent Carrier and Exciton Generations in Regioregular Poly(3-hexylthiophene) Polymer Diodes as Revealed by Bleaching Spectroscopy
}

Katsuichi Kanemoto, Motoaki Yasui, Daisuke Kosumi, Akihiko Ogata, Mitsuru Sugisaki, Tsutomu Karasawa, Ichiro Akai, Hideki Hashimoto

\begin{tabular}{|c|l|}
\hline Citation & Physical Review Letters, 103(18); 187402 \\
\hline Issue Date & 2009-10-30 \\
\hline Type & Journal Article \\
\hline Textversion & Publisher \\
\hline Rights & $\begin{array}{l}\text { This article may be downloaded for personal use only. Any other use requires prior } \\
\text { permission of the author and American Physical Society. } \\
\text { The following article appeared in Physical Review Letters Vol.103, Iss.18 and may } \\
\text { be found at https://doi.org/10.1103/PhysRevLett.103.187402 }\end{array}$ \\
\hline DOI & \begin{tabular}{l} 
10.1103/PhysRevLett.103.187402 \\
\hline
\end{tabular} \\
\hline
\end{tabular}

Self-Archiving by Author(s)

Placed on: Osaka City University

.Katsuichi Kanemoto. et al. (2009). Morphology-Dependent Carrier and Exciton Generations in Regioregular Poly(3-hexylthiophene) Polymer Diodes as Revealed by Bleaching Spectroscopy.

Physical Review Letters. 103. 187402. 


\title{
Morphology-Dependent Carrier and Exciton Generations in Regioregular Poly(3-hexylthiophene) Polymer Diodes as Revealed by Bleaching Spectroscopy
}

\author{
Katsuichi Kanemoto, ${ }^{1, *}$ Motoaki Yasui, ${ }^{1}$ Daisuke Kosumi, ${ }^{1}$ Akihiko Ogata, ${ }^{1}$ Mitsuru Sugisaki, ${ }^{1}$ Tsutomu Karasawa, ${ }^{1}$ \\ Ichiro Akai, ${ }^{2}$ and Hideki Hashimoto ${ }^{1}$ \\ ${ }^{1}$ CREST/JST and Department of Physics, Osaka City University, 3-3-138 Sugimoto, Sumiyoshi-ku, Osaka 558-8585, Japan \\ ${ }^{2}$ Shock Wave and Condensed Matter Research Center, Kumamoto University, 39-1 Kurokami 2, Kumamoto 860-8585, Japan
} (Received 22 April 2009; published 28 October 2009)

\begin{abstract}
Bleaching probe spectroscopy performed for regioregular poly(3-hexylthiophene) diodes reveals that coexistent morphological phases determine the conducting and optical properties of conjugated polymer films. Photoinduced absorption measurements demonstrate that exciton migration occurs from lamella aggregates to morphological sites consisting of quasiuncoupled chains and that the latter sites determine steady-state photophysical properties. Spectroscopy synchronized with diode operation reveals that the morphological locations of injected carriers in polymer diodes vary with the applied bias.
\end{abstract}

DOI: 10.1103/PhysRevLett.103.187402

Conjugated polymers are a remarkable class of material that combines prominent optical and electronic properties. This has enabled them to be used in optoelectronic devices such as light emitting diodes and photovoltaic devices $[1,2]$. Such remarkable properties are owing to excitons and carriers created by photoexcitation or bias impression. Elucidating the behavior of such excitations is thus the key to exploring the underlying physics of photoexcitation and device operations. Spectroscopic techniques have been used for investigating photoexcitations $[3,4]$ and carriers in polymer films and semiconducting devices [5-7].

The characteristic feature of polymers compared with inorganic or molecular semiconducting materials is the flexibility in the polymer chains and this imparts advantages in processing. This feature allows several morphological phases to coexist within a solid film. Hence, the physical properties of excitons and carriers in polymer films must essentially be evaluated with the consideration of their morphological location. However, such studies are difficult unless definite information on morphology is obtained like polyfluorene that has detectable absorption signals from additional morphological phases [8]. In this Letter, we attempt to elucidate the behaviors of excitons and carriers with respect to their morphological location in polymer films. We focus on the bleaching signals of absorption spectra induced by femtosecond-transient and continuous-wave (cw) photoexcitations or diode operation. Bleaching spectroscopy can probe the absorption spectrum of components consumed in certain physical events. We apply this technique because the absorption spectra of the consumed components can provide more straightforward information on the surrounding morphology of generated excitations than can direct spectral features of the excitations.

In this study, we use a polymer diode of head-to-tail coupled, regioregular (RR) poly(3-hexyllthiophene) (P3HT). RR-P3HT is known to form solid films that have self-organized microcrystalline domains $[9,10]$, whose
PACS numbers: 78.66.Qn, 72.80.Le, 73.61.Ph, 78.40.Me

property makes it a promising polymer for FET applications. We define the RR-P3HT film as a prototype of the aggregate phase that is partly present in normal polymer films consisting of randomly distributed chains. The RR-P3HT film, generally believed to have a well-defined solid structure, is shown to have several morphological phases that have different roles in optical and electronic applications. We further show that bleaching spectroscopy can elucidate the morphology-dependent behaviors of excitons and carriers under photoexcitation and diode operation. It is revealed that the correlation between the excitations and their morphological locations is a critical factor for describing the photophysical and conducting properties of conjugated polymer films.

RR-P3HT powder was purchased from Aldrich and used as received. A diode used in optical measurements consisted of four-layers: an indium-tin-oxide (ITO) coated glass substrate, a hole-transporting PEDOT-PSS layer (hereafter PEDOT), the RR-P3HT film and a semitransparent $\mathrm{Al}$ electrode deposited by vacuum evaporation. The RR-P3HT film was fabricated by spin-casting its chlorobenzene solution at a concentration of $10 \mathrm{mg} / \mathrm{mL}$. The combined thickness of the PEDOT and RR-P3HT layers was about $100 \mathrm{~nm}$.

Photoinduced absorption (PIA) measurements were performed under $\mathrm{cw}$ or pulsed photoexcitation. The diode sample was used for both PIA measurements, but observed spectral features were not different from those of film samples with no electrodes. For the cw-PIA measurement, excitation was provided by an $\mathrm{Ar}^{+}$laser at $488 \mathrm{~nm}$ $(\sim 50 \mathrm{~mW})$ modulated by an acoustic-optic modulator at $700 \mathrm{~Hz}$. The probe beam for the measurement was produced using a tungsten/halogen lamp. The cw-PIA signals were detected by a phase-sensitive lock-in technique with a monochromator and a Si photodiode. Photoluminescence (PL) signals were eliminated by subtracting a spectrum measured without a probe beam. The laser system used for the transient PIA measurement consisted of a Ti:sapphire 
laser (Spectra Physics, Hurricane-X) with a repetition rate of $1 \mathrm{kHz}$ at $780 \mathrm{~nm}(\sim 100$ fs pulse duration). An optical parametric amplifier (OPA, Spectra Physics, OPA-800C) pumped by a portion of the Ti:sapphire beam was used to produce wavelength-tunable visible pump pulses that were tuned to $480 \mathrm{~nm}(50 \mathrm{~nJ} /$ pulse $)$. A white-light continuum produced in a sapphire plate by the amplified 780-nm beam was used as probe pulses. The probe pulses were dispersed using a spectrograph and imaged by a 1024-pixel linear photodiode array [11]. Absorption and bleaching signals induced by diode operation were measured by applying dc and ac voltages ( $V_{\mathrm{dc}}$ and $V_{\mathrm{ac}}$, respectively) to the diode device and detecting the modulated signal of the transmitted light synchronized with the ac voltage $(700 \mathrm{~Hz})$ (hereafter, referred as device modulation (DM) spectroscopy). The same probe system was used for detection as that used for the cw-PIA measurement. All of the optical measurements were performed at room temperature in an inert atmosphere.

Figure 1 shows the absorption and cw-PIA spectra of the RR-P3HT film. The PIA spectrum has absorption peaks at 1.25 and $1.85 \mathrm{eV}$. Similar features were previously reported and they were shown to be attributed to photogenerated polarons $[4,12]$. We thus consider that the observed peaks are due to the polarons. The PIA spectrum exhibits clear bleaching signals at energies above $1.87 \mathrm{eV}$. The bleaching signals correspond to the absorption spectrum of neutral species that are converted to polarons by photoexcitation. Interestingly, the bleaching spectrum clearly differs from the steady-state absorption spectrum shown in Fig. 1: the bleaching spectrum is redshifted, is much sharper, and has a different spectral pattern.

Figure 2 shows the transient PIA spectra of the RRP3HT film. The PIA spectra were recorded at 0, 10, 200 and 400 ps after excitation. They exhibit clear bleaching signals, while small absorption signals of photoexcitations are observed around $1.88 \mathrm{eV}$ in the spectra at 0 and $10 \mathrm{ps}$. The absorption signals are attributed to photogenerated polarons (polaron pairs) [12]. The bleaching signals have some interesting features: the spectra change a spectral pattern with time and gradually redshift. These observa-

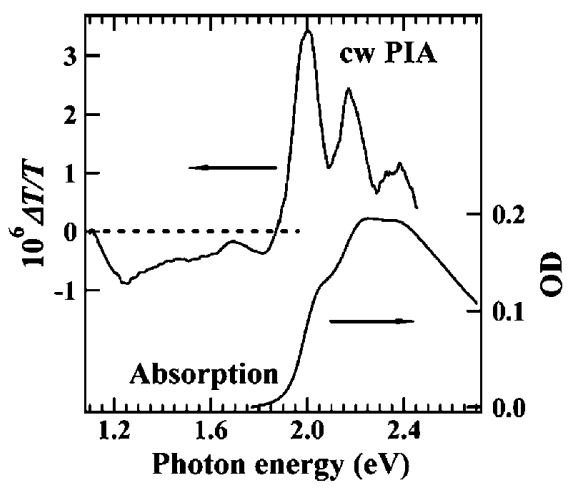

FIG. 1. Steady-state absorption and cw photoinduced absorption (PIA) spectra of the RR-P3HT film. The horizontal broken line is the baseline for the PIA spectrum. tions indicate that the neutral state converted to excitons or polarons gradually drops to a lower-energy state that has a different electronic structure. Related to these, the timeresolved PL spectra of the conjugated polymer films are known to redshift with time, and such a dynamical redshift has been ascribed to exciton migration [13-15]. An RR-type polythiophene derivative was also shown to exhibit dynamical redshift in PL [14] and the time scale of the PL redshift is similar to that which we observed. Therefore, we conclude that the observed time evolution of the bleaching spectra is due to exciton migration. The bleaching spectra hence correspond to the absorption spectra at sites where excitons are present, and their spectral variation indicates a picture of exciton migration between sites of different morphology. Some spectral differences are found between the $\mathrm{cw}$ and the 400-ps transient PIA bleaching spectra, as indicated by the broken line in Fig. 2 denoting the peak position of the $\mathrm{cw}$ one; the cw-PIA spectrum has lower-lying peaks and sharper spectral width. The cw-PIA bleaching spectrum can be regarded as being the absorption spectrum of the final migration point. The sharper width of the cw-PIA spectrum hence indicates that the location of the photoexcitations is finally restricted within specific domains.

Figures 3(a) and 3(b), respectively, show the DM spectra and the current-voltage $(I-V)$ characteristics for the RR$\mathrm{P} 3 \mathrm{HT}$ diode. The $I-V$ characteristics show that the current increases linearly in the low voltage region and then rises rapidly at $1 \mathrm{~V}$. This type of crossover has often been observed in the $I-V$ curves of organic insulators and the rapid rise is a typical behavior of the space-charge-limited current (SCLC) [16]. The DM spectra were recorded under biases of $V_{\mathrm{dc}}+V_{\mathrm{ac}}=0 \pm 1,0.7 \pm 1$, and $1 \pm 1 \mathrm{~V}$. The observed spectra in Fig. 3(a) exhibit some features of both the absorption and bleaching regions. However, when evaluating the effect of applying a bias on the spectrum,

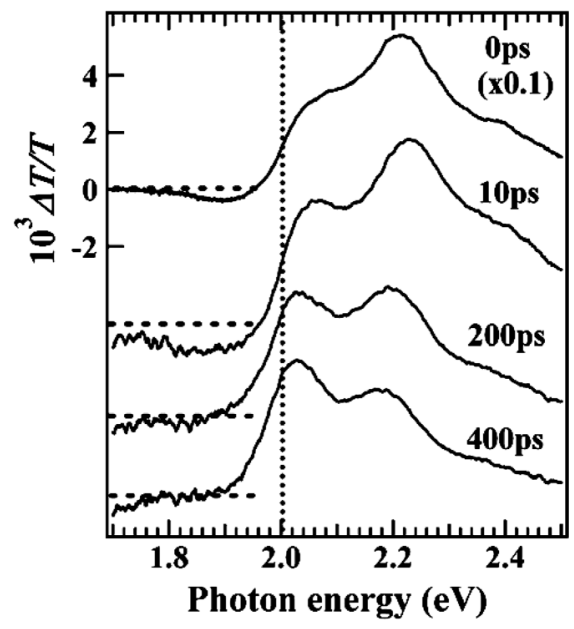

FIG. 2. Transient PIA spectra of the RR-P3HT film recorded at $0,10,200$, and $400 \mathrm{ps}$. The horizontal broken lines are the baselines for each spectrum and the vertical dotted line indicates the energy position of the $0-0$ peak in the cw-PIA spectrum. 


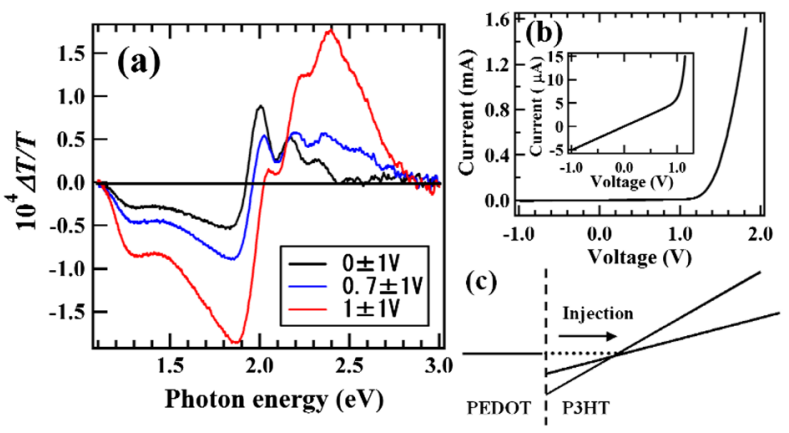

FIG. 3 (color online). (a) DM spectra under $V_{\mathrm{dc}}+V_{\mathrm{ac}}=0 \pm$ $1,0.7 \pm 1$, and $1 \pm 1 \mathrm{~V}$ and (b) the current-voltage characteristics for the RR-P3HT diode. (c) Schematic of hole injection by tunneling from PEDOT to two neutral states with different energy levels in P3HT. The two solid lines with different slopes in P3HT represent the difference of applied bias necessary for charge injection.

the contribution of electroabsorption (EA) to bleaching spectra has to be carefully considered because it could dominate the bias effect in the case of insufficient carrier injection $[17,18]$. The EA signals are known to be independent of the frequency of the ac voltage [19]. The difference of DM spectrum between different frequency measurements can hence eliminate the EA signals and it resembled the DM spectra shown in Fig. 3(a) [20]. This indicates that the EA contribution is negligible in the observed DM spectra. Indeed, the bleaching spectrum at $0 \pm 1 \mathrm{~V}$ resembles the bleaching spectrum obtained from the cw-PIA and the 400-ps transient PIA measurements, which were taken with no bias.

It was reported that the work functions of PEDOT and $\mathrm{Al}$ are $4.9 \mathrm{eV}[21,22]$ and $4.2 \mathrm{eV}$, respectively, and that the energy level of the highest occupied molecular orbitals (HOMO) of RR-P3HT is 5.1-5.2 eV [23,24]. The RR-P3HT diode used in this study can thus be regarded as a hole-only device. Hence, the features of the absorption region around 1.30 and $1.85 \mathrm{eV}$ in the three $\mathrm{DM}$ spectra are attributed to injected positive polarons that work as trapped or mobile carriers. The intensity of absorption signals is found to increase with the center bias, indicating that the number of the polarons increases with the applied voltage in the SCLC regime. This indicates a signature of SCLC that is enhanced by a voltage-induced increase in the carrier number [16].

The DM spectra exhibit remarkable features; the bleaching signals blueshift and change a spectral pattern with an increase in the applied dc bias, while the absorption region exhibits no appreciable spectral variations. The bleaching signals in the DM spectra correspond to the absorption spectra of neutral components converted into positive polarons. The features in bleaching signals thus demonstrate that the converted neutral components vary with the bias and that the lower-energy components are predominantly consumed when carriers are generated under a lower bias. This behavior could be explained by assuming independent neutral components having different energy levels, as depicted in Fig. 3(c). Specifically, a component with lower transition energy is expected to have a lower threshold voltage for hole injection. This is caused by the coexistence of at least two independent neutral components with different threshold voltages. We thus expect that, in the case of normal amorphous polymer films, such a change of converted neutral components with the bias should occur more noticeably due to randomly distributed electronic states.

We have identified the presence of several bleaching components in the RR-P3HT diode, which can be roughly classified into three: species $A$, which is observed in the $\mathrm{cw}$-PIA measurement and the DM measurement under $0 \pm 1 \mathrm{~V}$, species $B$, which is observed in the DM measurement under $1 \pm 1 \mathrm{~V}$, and species $C$, which is observed in the transient PIA measurement at 0 ps. It has been documented that, although weak vibronic peaks in the absorption spectrum of the RR-P3HT film arise from its organized solid structures, the peak pattern cannot be reproduced by a simple Franck-Condon (FC) progression $[25,26]$; the reason for this has been debated in recent years [25-27]. We note that the sum of the bleaching spectra of species $B$ and $C$ is likely to resemble the absorption spectrum, although that of species $B$ is partly distorted due to signals in the absorption region. This indicates that the absorption spectrum does not consist of a single species and that species $A$ is a minor component in the film.

We have fit the bleaching spectra of species $A, B$, and $C$ with a FC model using a single Huang-Rhys (HR) factor. Figure 4 shows the results of fitting the cw-PIA spectrum (species $A$ ). The fitting demonstrates that the spectrum is well reproduced by a simple FC progression; the HR factor, the 0-0 transition energy, and the phonon energy obtained by the fit are $0.745,2.000 \mathrm{eV}$, and $0.184 \mathrm{eV}$, respectively. By contrast, the same analysis did not reproduce well the bleaching spectra of species $B$ and $C$. It has been reported that the lamella aggregates of RR-P3HT give a PL spectrum whose $0-0$ transition is forbidden due to interchain couplings unless disorder is present [27]. This

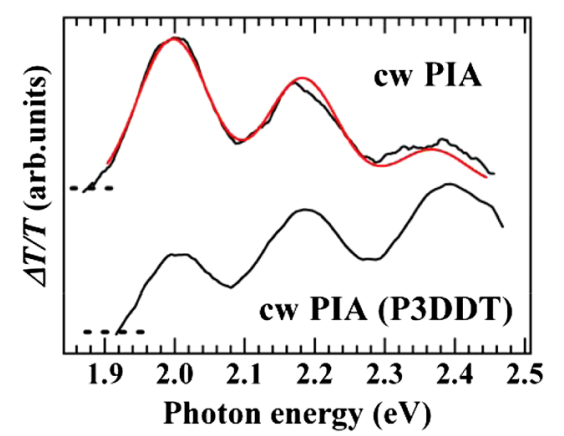

FIG. 4 (color online). Bleaching spectra obtained from the cwPIA measurements for RR-P3HT and RR-P3DDT films. The horizontal broken lines in the spectra are the baselines for each spectrum. The red solid curve for the spectra of RR-P3HT is a result of spectral simulation by the Franck-Condon model. 
feature is considered to cause the 0-0 transition of absorption also to decrease, so that the absorption spectrum cannot be reproduced by a FC progression. This suggests that species $B$ and $C$ are lamella aggregates.

By contrast, species $A$ may not be a lamella aggregate since its bleaching spectrum is reproduced by a single HR factor. Optical spectra reproduced by a single HR factor have been hardly reported on polymer samples except for the PL spectra of diluted polymers and long oligomers $[28,29]$. One might thus consider that species $A$ arises from isolated chains. Figure 4 shows the bleaching spectrum of an RR-poly(3-dodecylthiophene) (P3DDT) film obtained from cw-PIA measurements at room temperature. It demonstrates that the RR-P3HT and P3DDT films have clearly different bleaching spectra. A notable difference between the polymers is the length of the alkyl substituents, resulting in different interlamella distances in the solid state [16.36 $\AA$ (P3HT) and 27.19 $\AA$ (P3DDT)] [10]. This indicates that species $A$ is stabilized by the reduced interlamella distance in P3HT, leading to the conclusion that species $A$ should not arise from isolated chains. Alternatively, species $A$ could be a quasi-intrachain species created at certain aggregates. We consider that the reduced interlamella distance in P3HT induces slight deformation of the lamella structure or weakens interchain couplings due to created interlamella couplings and that it causes such intrachain states to create.

We finally describe the behaviors of excitons and carriers with their morphological locations by evaluating the observed bleaching spectra. The transient PIA spectra showed that photoexcitation commences with the consumption of species $C$. Considering the coexistence of several morphological components, other components may also be photoexcited as long as they have an oscillator strength at $480 \mathrm{~nm}$. We note that photoexcitations at 460 and $500 \mathrm{~nm}$ also exhibited species $C$ at the 0 ps bleaching spectrum. This indicates that species $C$ always functions as a starting point for photoexcitation. This would be because species $C$ has a higher oscillator strength than others. Photoexcitation is followed by migration of excitons and the excitons finally reach the quasiuncoupled chain sites (species $A$ ). This indicates that steady-state optical properties such as PL and photoconductivity are determined by excitations at the morphological sites of species $A$.

The DM measurements revealed that carriers are generated at the uncoupled chain sites at low voltages. By contrast, at typical operational biases that are greater than the build-up voltage, carriers are generated at lamella aggregates (species $B$ ). This indicates that carriers are injected at different morphological sites depending on the applied bias. This suggests that the carrier locations can be controlled by varying the applied bias in the RR-P3HT diode. In the diode operation, primary carriers are provided by species $B$, which was not detected in the PIA measurements. We thus emphasize that different morphological components are responsible for conducting and optical properties in the $\mathrm{P} 3 \mathrm{HT}$ diode.
In summary, bleaching spectroscopy demonstrated that the RR-P3HT film has several morphological phases that have different roles in optical and electronic applications. Transient absorption measurements revealed exciton migration occurring between different morphological sites. The nature of the final migration point that determines the steady-state photophysical properties was revealed. DM spectra of the P3HT diode showed that the morphological sites at which carriers are created vary with the applied bias. Our results emphasize that the optical and conducting properties of the polymers may not be explained by a simple model assuming single kind of excitations.

This work was supported in part by funding from the CASIO Science Promotion Foundation.

*Corresponding author.

kkane@sci.osaka-cu.ac.jp

[1] R. H. Friend et al., Nature (London) 397, 121 (1999).

[2] S. Günes, H. Neugebauer, and N. S. Sariciftci, Chem. Rev. 107, 1324 (2007).

[3] Z. V. Vardeny and X. Wei, in Handbook of Conducting Polymers II, edited by T. A. Skotheim, R. L. Elsenbaumer, and J. R. Reynolds (Marcel Dekker, New York, 1997).

[4] R. Österbacka et al., Science 287, 839 (2000).

[5] H. Sirringhaus et al., Nature (London) 401, 685 (1999).

[6] P. J. Brown et al., Phys. Rev. B 63, 125204 (2001).

[7] A. S. Dhoot et al., Chem. Phys. Lett. 360, 195 (2002).

[8] M. Grell et al., Macromolecules 32, 5810 (1999).

[9] R. D. McCullough et al., J. Am. Chem. Soc. 115, 4910 (1993).

[10] T. A. Chen, X. M. Wu, and R. D. Rieke, J. Am. Chem. Soc. 117, 233 (1995).

[11] D. Polli et al., Chem. Phys. 350, 45 (2008).

[12] O. J. Korovyanko et al., Phys. Rev. B 64, 235122 (2001).

[13] R. Kersting et al., Phys. Rev. Lett. 70, 3820 (1993).

[14] T. Kobayashi et al., Phys. Rev. B 62, 8580 (2000).

[15] K. Kanemoto et al., J. Phys. Chem. B 111, 12389 (2007).

[16] M. A. Lampert and P. Mark, Current Injection in Solids (Academic Press, New York, 1970).

[17] M. Liess, Z. V. Vardeny, and P. A. Lane, Phys. Rev. B 59, 11053 (1999).

[18] I. H. Cambell et al., Phys. Rev. Lett. 76, 1900 (1996).

[19] P. A. Lane et al., Appl. Phys. Lett. 83, 3611 (2003).

[20] See EPAPS Document No. E-PRLTAO-103-044946. For more information on EPAPS, see http://www.aip.org/ pubservs/epaps.html.

[21] G. Winroth et al., Appl. Phys. Lett. 92, 103308 (2008).

[22] F. Petraki, S. Kennou, and S. Nespurek, J. Appl. Phys. 103, 033710 (2008).

[23] M. Onoda et al., Thin Solid Films 331, 76 (1998).

[24] R. Valaski et al., J. Appl. Phys. 92, 2035 (2002).

[25] P. J. Brown et al., Phys. Rev. B 67, 064203 (2003).

[26] J. Clark et al., Phys. Rev. Lett. 98, 206406 (2007).

[27] F. C. Spano, J. Chem. Phys. 122, 234701 (2005); 126, 159901 (2007).

[28] K. Kanemoto et al., Phys. Rev. B 73, 235203 (2006).

[29] K. Kanemoto et al., J. Chem. Phys. 130, 234909 (2009). 